\title{
Medical Fashion Victims? Do Nail Polishes and Acrylic Nails affect digital pulse oximetry and patient management in the clinical setting?
}

\section{J. Purcell' 1 , S. Mannion² \\ ${ }^{1}$ University College Cork - Cork (Ireland), ${ }^{2}$ South Infirmary Victoria University Hospital - Cork (Ireland)}

Background and Goal of Study:

Digital Pulse Oximetry (DPO) is ubiquitous in monitoring patients' oxygen saturation $\left(\mathrm{SpO}_{2}\right)$ levels. Concerns prevail that nail treatments such as acrylic nails or nail polishes affect these readings, but published data is inconsistent. As changes in $\mathrm{SpO}_{2}$ levels affect the levels of interventions and impact patient monitoring in critical care and anaesthesia, discerning if nail treatments adversely affect DPO is crucial.

1: Survey healthcare staff attitudes and approaches to this issue

2: Experimentally assess effects of specific nail treatments on $\mathrm{SpO}_{2}$ readings under different physiological conditions

\section{Materials and Methods:}

1: Questionnaire based survey was issued to clinical staff at four university hospitals to assess current knowledge and opinions regarding how nail treatments impact on clinical decisions pertaining to DPO.

2:An interventional experiment on 12 volunteers analysing effects of nail polish of different colours and acrylic nails from popular brands, on $\mathrm{SpO}_{2}$ measurements under varying physiological conditions (healthy, venous congestion, venous constriction).

Nail varnish and fake/acrylic nails are often considered to interfere with patient pulse oximetry readings. Does this consideration interfere with your practice?

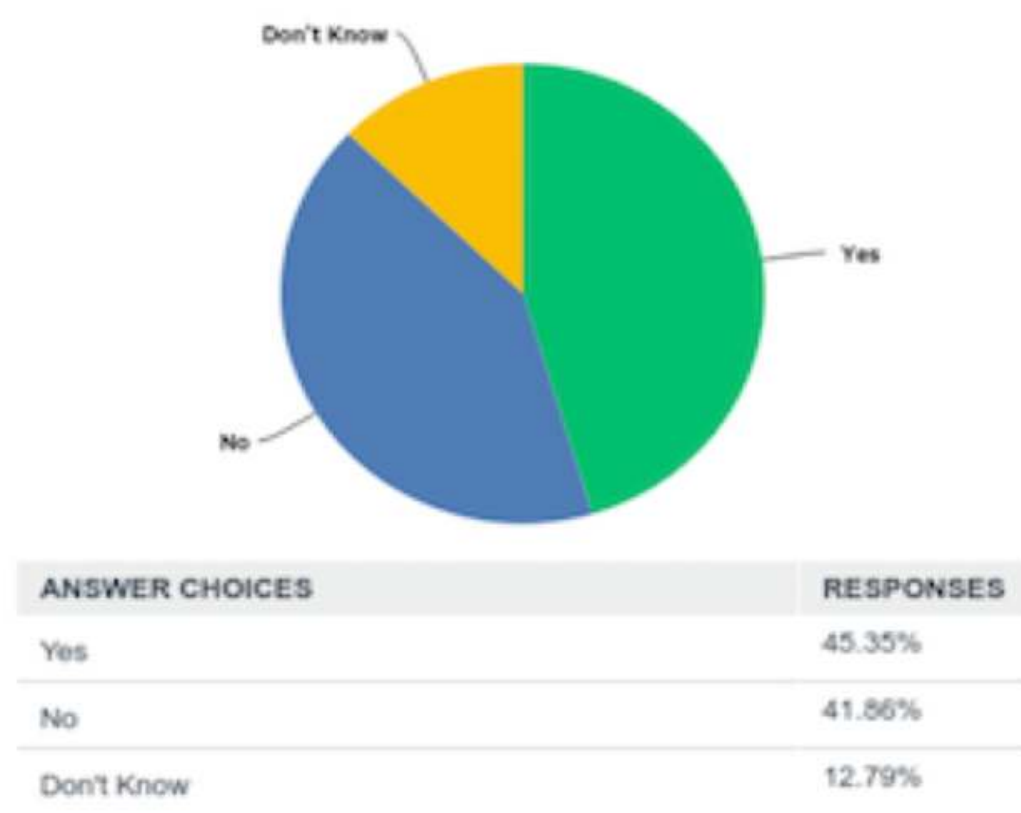

Congestion was modelled using venous stasis on noninvasive blood pressure cuffs and constriction was modelled using water immersion for $10 \mathrm{~min}$ at $10^{\circ} \mathrm{C}$.

\section{Results and Discussion:}

Survey: 86 Respondents (55 doctors, 21 nurses). Nail treatments affect $45 \%$ of respondents' clinical practice, and $>30 \%$ of respondents intervene to remove treatments.

Experiment $(\mathrm{n}=12): \Delta<1 \%( \pm$ SEM $)$ in $\mathrm{SpO}_{2}$ readings across all nail treatments and models analysed, none of which resulted in a $\mathrm{SpO}_{2}$ reading $<95 \%$, at which $\mathrm{O}_{2}$ intervention is recommended.

Conclusions: Knowledge of, and approach to, potential complications of nail treatments on DPO varies amongst healthcare staff, with poor knowledge or understanding of effects. Our survey data suggest it would be reasonable to establish hospital policies that do not require prior removal of nail treatments examined in this study.

Experimental data indicate the nail treatments specified do not contribute significantly to a difference in SpO2 readings, therefore have no clinical impact on patient care.

Do you believe that nail varnish colour has an impact on the readings given by the device?

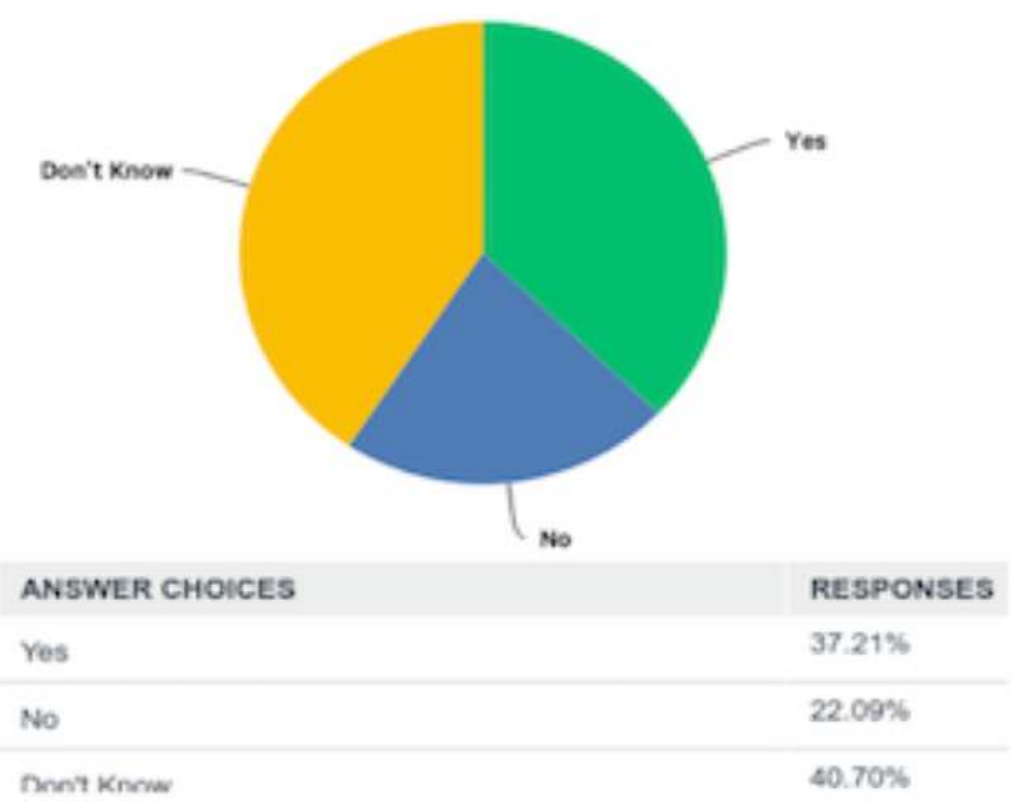

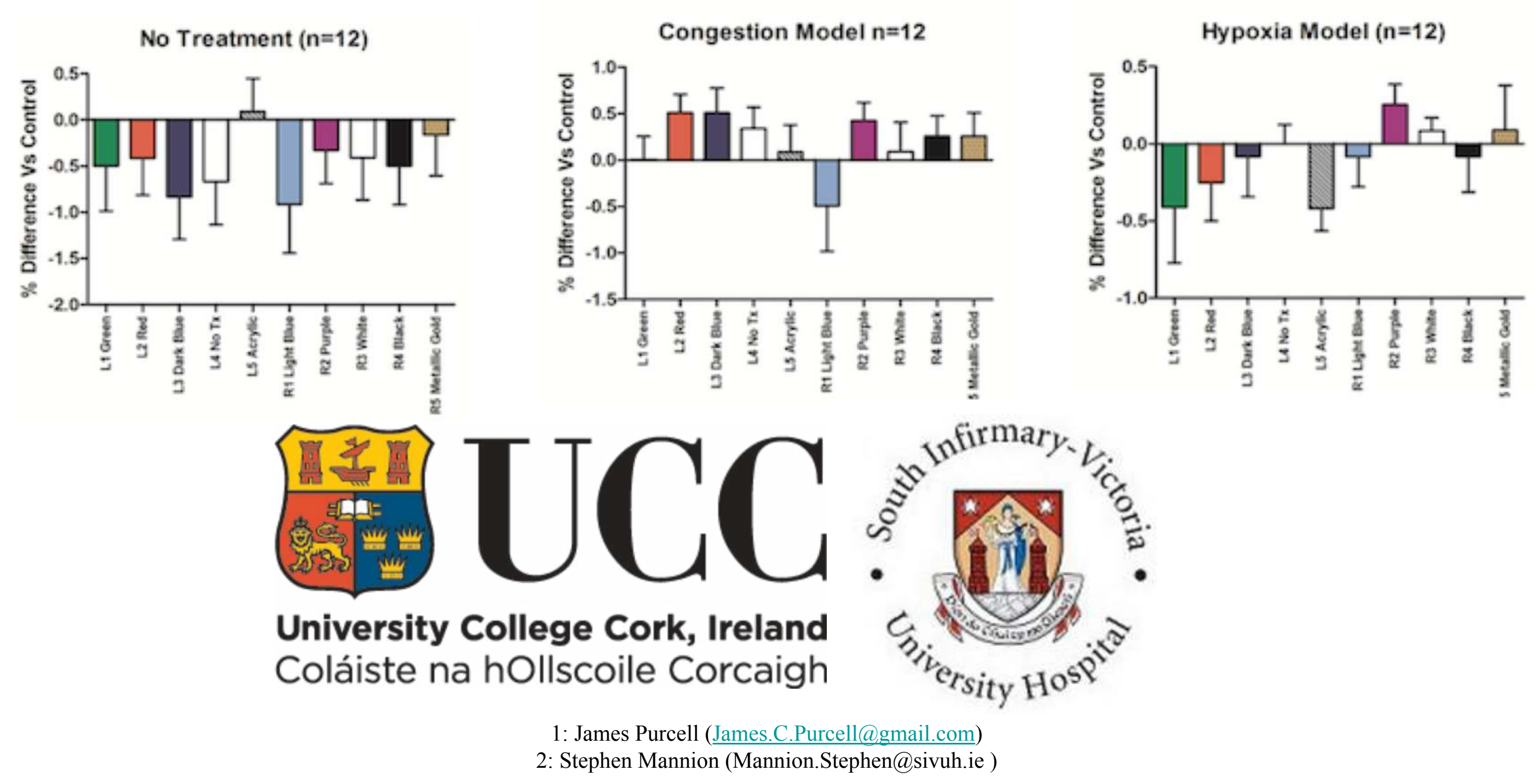

